\title{
Milia may originate from the outermost layers of the hair bulge of the outer root sheath: A case report
}

\author{
ICHIRO KUROKAWA $^{1}$, AYAKO KAKUNO ${ }^{2}$ and AIRO TSUBURA ${ }^{3}$ \\ ${ }^{1}$ Department of Dermatology, Acne Clinical Research Center; ${ }^{2}$ Department of Pathology, Meiwa Hospital, Nishinomiya, \\ Hyōgo 663-8186; ${ }^{3}$ Department of Pathology, Kansai Medical University, Moriguchi, Osaka 570-8506, Japan
}

Received January 12, 2016; Accepted September 1, 2016

DOI: $10.3892 / \mathrm{ol} .2016 .5335$

\begin{abstract}
It has been hypothesized that milia originate from the hair bulge of the outer root sheath. To elucidate the histogenesis of milia, an immunohistochemical study was performed using anti-keratin and anti-filaggrin antibodies to determine the levels of keratin and filaggrin expression. Keratin expression was evaluated using anti-keratin antibodies against K1, K7, K8, K10, K14, K15, K16, K17, K18, K19 and K20. K1 and K10 expression were detected in the suprabasal layers of the more superficial section of the cyst walls, but not in the deeper section of the cyst walls. However, K14 and K17 were expressed in all layers of the cyst walls. Notably, K15 was expressed in the outermost layer of the deeper section of the cyst walls and hair germ structure, whereas filaggrin was expressed in the superficial layer of the more superficial section of the cyst walls. Therefore, the pattern of keratin and filaggrin expression indicates that milia may originate from the outermost cells of the hair bulge of the outer root sheath.
\end{abstract}

\section{Introduction}

Milia appear on the face as multiple white globoid papules, and is usually treated by evacuation with a comedone extractor (1). It has been suggested that milia may be derived from the lowest portion of the infundibulum of vellus hairs at the level of the sebaceous duct (2). However, the histogenesis and origin of milia remain unclear. Milia typically appear on the face, particularly in aged women, and may be formed by an obstruction of the follicular infundibulum (1). A previous study demonstrated that keratin, particularly K1, K10, K14 and K16, is expressed in milia (3). In addition, it has been reported that milia occur due to the cystic alteration of trichoepitheliomatous bulge proliferation (4). In experimental autotransplantation, milia may attach to the outer root sheath near the insertion

Correspondence to: Dr Ichiro Kurokawa, Department of Dermatology, Acne Clinical Research Center, Meiwa Hospital, 4-31 Agenaruo-cho, Nishinomiya, Hyōgo 663-8186, Japan

E-mail: kurokawa.i@meiwa-hospital.com

Key words: milia, hair bulge, keratin point of the arrector pili muscle (2). Therefore, milia may be originated from hair bulge of the outer root sheath. The aim of our study is to identify the origin of milia with immunohistochemical methods using keratin and filaggrin.

Keratin is a useful marker to evaluate the origin of epithelial tumors and filaggrin is a marker of terminal differentiation (5). In normal hair follicles, $\mathrm{K} 10$ and $\mathrm{K} 1$ are expressed in the infundibulum and suprabasal epidermis (5). K14 is expressed in all layers of the outer root sheath and infundibulum and in the basal layer of the epidermis. K15 is expressed in the outermost cells of the hair bulge, while K16 is expressed in the outer root sheath beneath the opening of the sebaceous duct (5). K17 is expressed in all layers of the outer root sheath and in the infra-infundibulum (5). By contrast, filaggrin is expressed in the superficial layer of the infundibulum (5). Therefore, it was hypothesized that milia may originate from the hair bulge of outer root sheath. To elucidate the histogenesis of milia, immunohistochemistry was performed using anti-keratin and anti-filaggrin antibodies to determine the expression of keratin and filaggrin.

\section{Case report}

A 74-year-old woman presented to the Department of Dermatology, Meiwa Hospital (Nishinomiya, Japan) on May 2015 with multiple white papules on the face, which had persisted for 10 years (Fig. 1). Milia were diagnosed by the clinical feature of facial white papules.

The skin biopsy was obtained from a white papule under local anesthesia. The specimens were fixed with formalin, embedded in paraffin, and cut into sections of 5-7- $\mu \mathrm{m}$ thickness. Selected sections were stained with hematoxylin and eosin. Analysis of a skin biopsy resulted in the diagnosis of primary milia, with hematoxylin and eosin staining indicating the presence of dilated follicular cysts. The epithelial lining of the cyst walls consisted of two sections: The more superficial and the deeper sections. Keratohyalin granules were detected in the more superficial section of the epithelial lining, but were not detected in the deeper section. Hair germ structures were identified in the deeper section of the epithelial lining.

On immunohistochemical examination for keratin and filaggrin expression, the labeled streptavidin-biotin (LSAB) method was used (Dako North America, Inc., Carpinteria, CA, USA). The following mouse anti-human keratin antibodies 


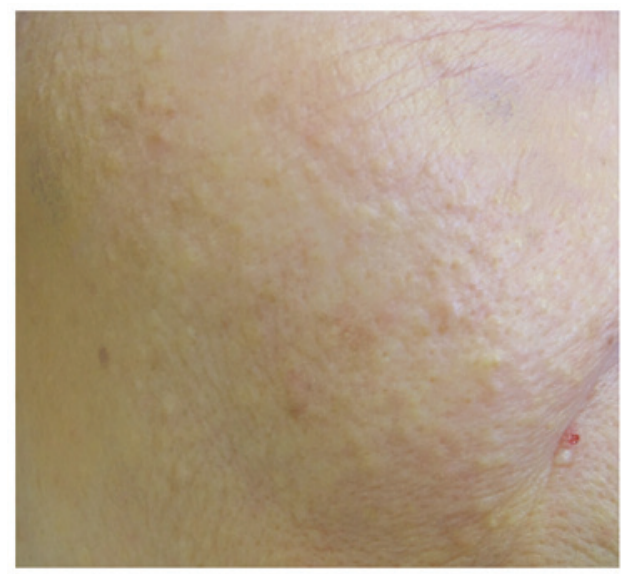

Figure 1. Multiple whitish papules on the face of the patient were observed clinically.

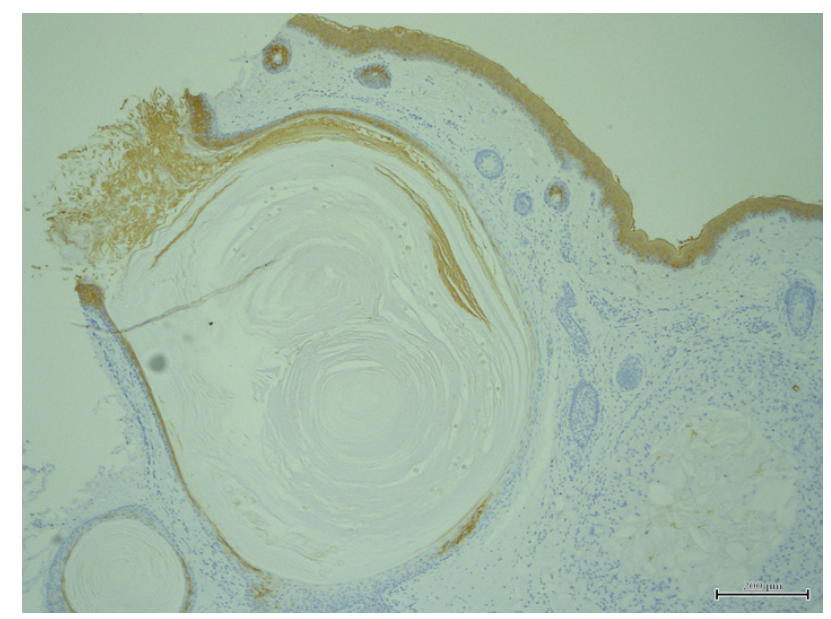

Figure 2. K1 was expression was detected in the suprabasal layers of the more superficial section of the cyst walls. However, K1 expression was attenuated in the deeper section of the cyst walls (original magnification, $\mathrm{x} 4$ ).

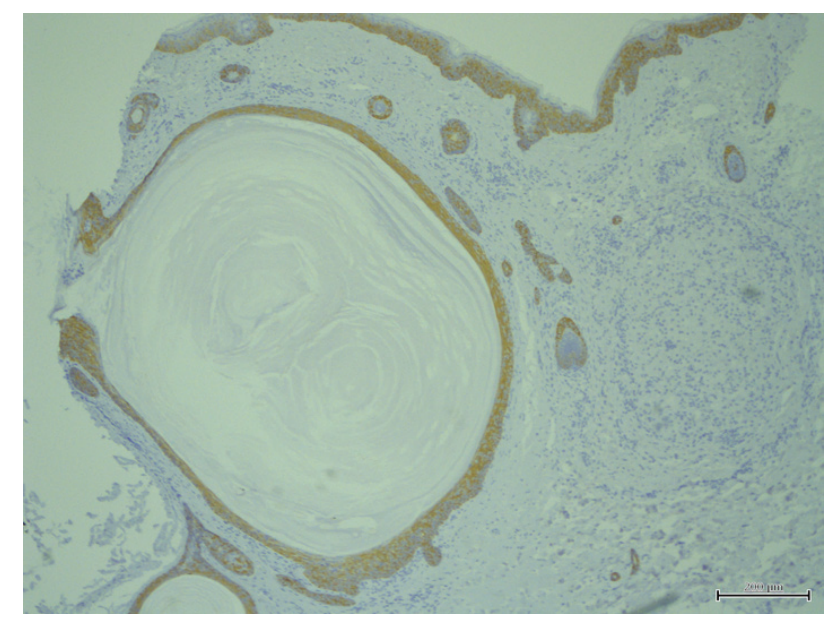

Figure 3. K14 was expressed in all layers of the cyst walls (original magnification, $\mathrm{x} 4)$.

were used in the present study: 34ßB4 (anti-K1; dilution 1:50), LP5K (anti-K7; dilution 1:10), LP3K (anti-K8; dilution 1:50), HP1 (anti-K10; dilution 1:50), LL002 (anti-K14; dilution

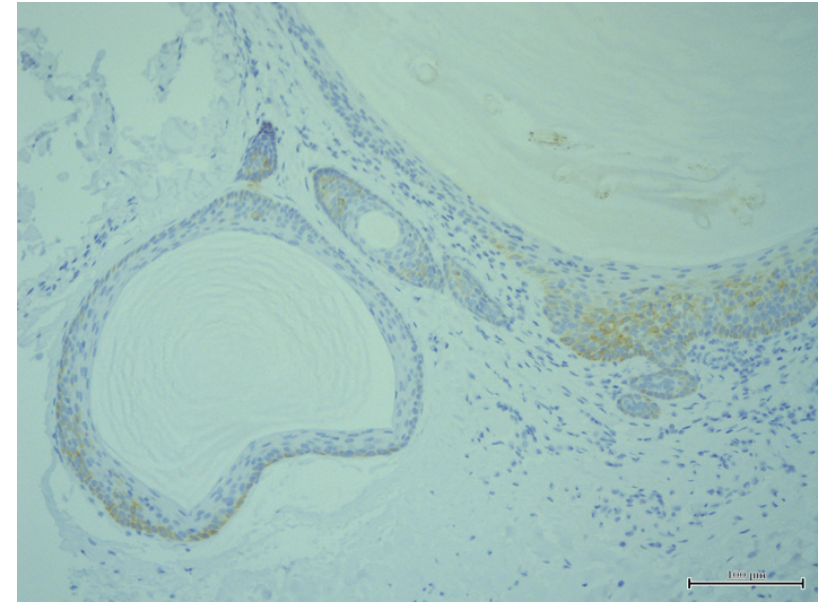

Figure 4. K15 was expressed in the outermost layer of the deeper section of the cyst walls and hair germ structure (original magnification, x20).

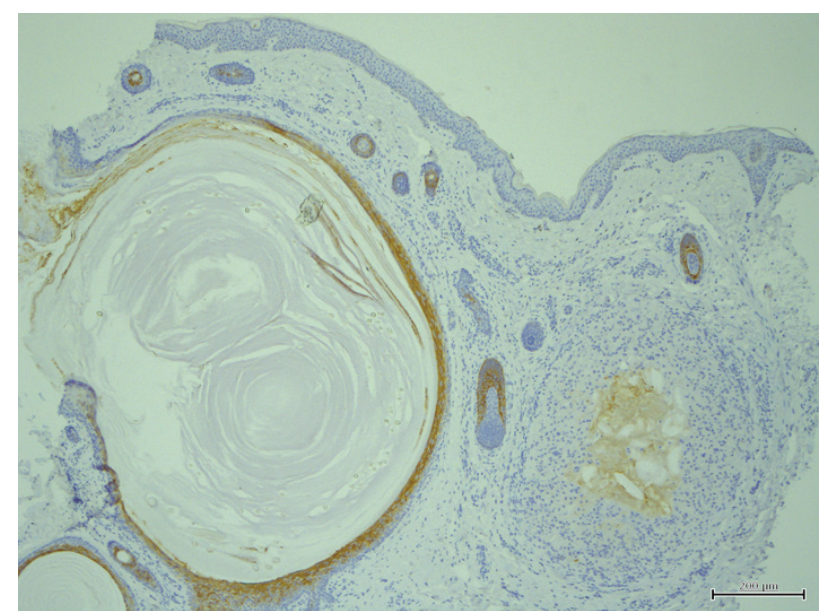

Figure 5. K17 was expressed in all layers of the cyst walls (original magnification, $\mathrm{x} 4)$.

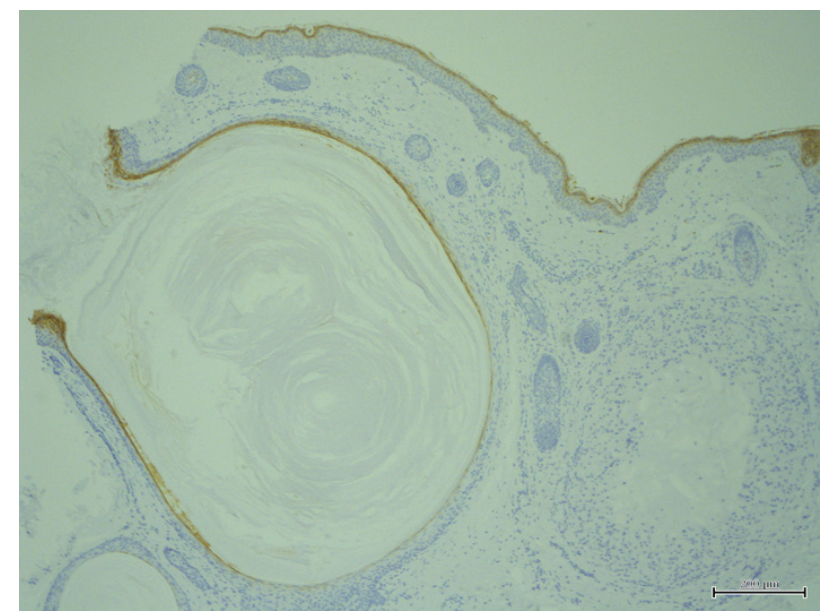

Figure 6. Filaggrin was expressed in the superficial layer of the more superficial section of the cyst walls (original magnification, $\mathrm{x} 4$ ).

1:200), LHK15 (anti-K15; dilution 1:40), LL025 (anti-K16; dilution 1:20), E3 (anti-K17; dilution 1:25), 5D3 (anti-K18; dilution 1:20), b170 (anti-K19; dilution 1:100), Ks 20.8 
(anti-K20; dilution 1:25), and 15C10 (anti-filaggrin; dilution 1:50), all from Novocastra Laboratories Ltd. (New castle upon Tyne, UK). The LSAB method was applied according to the manufacturer's protocol. The levels of keratin expression were assessed using anti-keratin antibodies against K1, K7, K8, K10, K14, K15, K16, K17, K18, K19, K20 and filaggrin. K1 (Fig. 2) and K10 expression was detected in the suprabasal layers of the more superficial section of the cyst walls and their expression was attenuated in the deeper sections. By contrast, K14 expression was observed in all layers of the cyst walls (Fig. 3). Notably, K15 was expressed in the outermost layer of the deeper area of the cyst walls and hair germ structures (Fig. 4), while K17 was expressed in all layers of the cyst walls (Fig. 5). The expression of other keratins was not detected in the cyst walls. Filaggrin was expressed in the superficial layer of the more superficial sections of the cyst walls (Fig. 6). The milia were treated with adapalene. However, no remarkable change was observed and the treatment was discontinued.

\section{Discussion}

Milia are derived from the lowest portion of the infundibulum of vellus hairs at the level of the sebaceous duct (1). The current study demonstrated that $\mathrm{K} 1$ and $\mathrm{K} 10$ were expressed in the more superficial sections of the cyst walls, and that filaggrin expression was detected in the more superficial part of the cyst walls, while K15 and K17 were expressed in the deeper sections of the cyst walls. K14 expression was detected in all cyst wall layers. In addition, it was observed that K16 was not expressed in milia. This is different from the results of a previous study (3) and may be explained by the diversified heterogeneity of milia differentiation. It is possible that the present case may not be a conventional example of milia and that individual differences between various cases of milia may exist.

Regarding the previously described pattern of keratin expression in normal hair follicles (5), the current study revealed that keratin expression in the more superficial section of the cyst wall is the same as that of the infundibulum, and that its expression in the deeper section is the same as that of the hair bulge of the outer root sheath. These results suggested that keratin and filaggrin expression in milia correspond to the more superficial section of the infundibulum and the outermost layer of the hair bulge, respectively. Furthermore, $\mathrm{K} 15$, a stem pluripotent cell marker, is expressed in the outermost layer in normal hair follicles (5). K15 was present in the outermost layer of the deeper section of cyst walls and hair germ structures in the present study. In trichoepithelioma, K15 expression is observed (6). A previous study demonstrated that milia were the result of cystic alteration of the trichoepitheliomatous bulge proliferation (4).

In conclusion, the present study reported a case of milia with an immunohistological evaluation of keratin and filaggrin expression. According to the pattern of keratin and filaggrin expression observed in the current study, milia may originate from the outermost cells of the hair bulge of the outer root sheath. Further studies concerning keratin and filaggrin expression in milia are necessary to confirm this hypothesis.

\section{References}

1. Berk DR and Bayliss SJ: Milia: A review and classification. J Am Acad Dermatol 59: 1050-1063, 2008.

2. Epstein W and Kligman AM: The pathogenesis of milia and benign tumors of the skin. J Invest Dermatol 26: 1-11, 1956.

3. Broekaert D, Leigh IM, Lane EB, Van Muijen GN, Ramaekers FC, De Bersaques J and Coucke P: An immunohistochemical and histochemical study of cytokeratin, involucrin and transglutaminase in seborrhoeic keratosis. Arch Dermatol Res 285: 482-490, 1993.

4. van der Putte SC: The pathogenesis of familial multiple cylindromas, trichoepitheliomas, milia, and spiradenomas. Am J Dermatopathol 17: 271-280, 1995.

5. Kurokawa I, Takahashi K, Moll I and Moll R: Expression of keratins in cutaneous epithelial tumors and related disorders-distribution and clinical significance. Exp Dermatol 20: 217-228, 2011.

6. Kanitakis J, Bourchany D, Faure M and Claudy A: Expression of the hair stem cell-specific keratin 15 in pilar tumors of the skin. Eur J Dermatol 9: 363-365, 1999. 